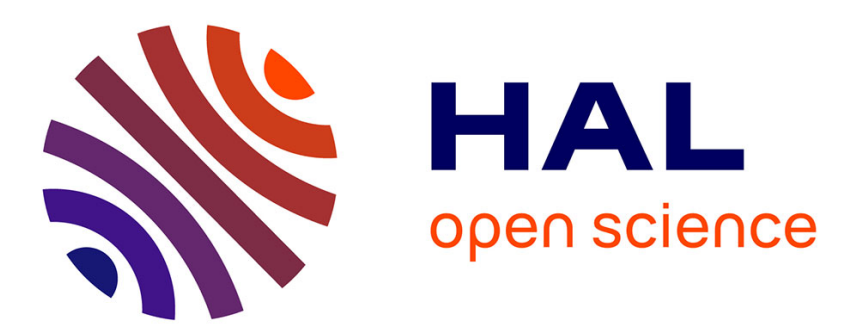

\title{
Comparative study between EMTR technique and a GA-based method for modeling EM radiation source in the Near Field
}

Sassia Hedia, Bassem Zitouna, Jaleleddine Ben Hadj Slama, Lionel Pichon

\section{> To cite this version:}

Sassia Hedia, Bassem Zitouna, Jaleleddine Ben Hadj Slama, Lionel Pichon. Comparative study between EMTR technique and a GA-based method for modeling EM radiation source in the Near Field. EMC Europe, Sep 2019, Barcelone, Spain. 10.1109/EMCEurope.2019.8872044 hal-02333935

\section{HAL Id: hal-02333935 \\ https://hal.science/hal-02333935}

Submitted on 23 Aug 2020

HAL is a multi-disciplinary open access archive for the deposit and dissemination of scientific research documents, whether they are published or not. The documents may come from teaching and research institutions in France or abroad, or from public or private research centers.
L'archive ouverte pluridisciplinaire HAL, est destinée au dépôt et à la diffusion de documents scientifiques de niveau recherche, publiés ou non, émanant des établissements d'enseignement et de recherche français ou étrangers, des laboratoires publics ou privés. 


\section{Comparative study between EMTR technique and a GA-based method for modeling EM radiation source in the Near Field}

\author{
Sassia HEDIA \\ ${ }^{1}$ Université de Sousse, Ecole Nationale \\ d'Ingénieurs de Sousse, LATIS- \\ Laboratory of Advanced Technology and \\ Intelligent Systems, 4023, Sousse, Tunisie \\ ${ }^{2}$ Group of Electrical Engineering of Paris, \\ CNRS UMR 8507, University of Paris-Sud, \\ University of Paris-Saclay, France \\ sassia.hedia@u-psud.fr
}

\author{
Bessem ZITOUNA, \\ Jaleleddine BEN HADJ SLAMA \\ ${ }^{1}$ Université de Sousse, Ecole Nationale \\ d'Ingénieurs de Sousse, LATIS- \\ Laboratory of Advanced Technology and \\ Intelligent Systems, 4023, Sousse, \\ Tunisie \\ bessem.zitouna@yahoo.fr \\ bhslama@yahoo.fr
}

\author{
Lionel PICHON \\ ${ }^{2}$ Group of Electrical Engineering, Paris \\ (GeePs), CNRS UMR 8507, Centrale \\ Supélec, University of Paris-Sud, \\ University of Paris-Saclay, France \\ Lionel.Pichon@supelec.fr
}

\begin{abstract}
This paper presents a comparative study between two different methods used in modeling electromagnetic transient disturbances of power electronic systems. The first method is the Electromagnetic Time Reversal (EMTR) technique based on time domain analysis. The second one is the frequency inverse method based on Genetic Algorithms (GA). The two methods are using the near field scanning technique. Moreover, both algorithms are established on the resolution of an inverse problem and they employ magnitude-only data. The obtained equivalent radiation models are compared with initial pattern for both simulation and experimental test cases. The frequency-time evaluation is discussed. The comparison between the two proposed methods shows that the EMTD method is more suitable for studying power electronics radiation and that it can provide an accurate equivalent model in a reduced time.
\end{abstract}

Keywords-EMC, Inverse Method, Near Field technique, EMTR, GA.

\section{INTRODUCTION}

A fast and accurate electromagnetic (EM) characterization is an essential task in the design stage of advanced power electronic systems. In such devices, radiation phenomena may cause electromagnetic interferences. Therefore, it is necessary to identify radiating sources and predict their emissions in the neighboring environment. A common proposed approach is the inverse problem resolution based on the Near Field (NF) scanning technique [1-10] [15-16]. This methodology provides an equivalent model that reproduces the same radiation pattern as the studied systems.

Several research works have been developed in order to obtain an accurate radiation model using equivalent magnetic elementary dipoles. The EM inverse problem has been established in the Frequency Domain (FD) in order to evaluate disturbances at different frequency levels [1-9]. Authors in [1] and [2] have proposed a matrix inversion method. This method supposes that dipoles positions and number are already known in advance and it is processed to determine only the moments of dipoles. In addition, it is a time consuming method. To overcome these difficulties, stochastic optimization methods have been developed in other works to estimate dipole parameters [3-7], namely the positions and moments. Beghou et al. [3], Liu et al. [4], Saidi et al. [5] [6], and Zhao [7] have employed different optimization algorithms. In fact, the obtained equivalent model, for a given frequency, can be established using a reduced number of dipoles. As a matter of fact, the most commonly used FD method for sources identification is the Genetic Algorithms (GA).

Indeed, multiples works have already been conducted on the FD, but far less on the Time Domain (TD). By analogy to the FD, EM inverse problem has been developed in TD [8-11] [12-16]. The TD analysis of the EM NF emissions can be particularly interesting for transient radiations prediction. Moreover, some recently explored concepts, that propose to deal with EMC aspects, include the Electromagnetic Time Reversal (EMTR) technique [11-16]. We presented in [15-16] the EM inverse method based on EMTR, using the NF in the $\mathrm{TD}$, in order to determine the equivalent dipole parameters, such as geometry and moment signal. This approach is intended to provide an equivalent behavioural pattern to characterize radiating sources in transient disturbances.

In this paper, a comparative study between a classic GAbased method and an EMTR-based method is presented. Section II is dedicated to theoretical investigations. In particular, both EM modelling approaches and their corresponding principles are studied. In section III, both methods are implemented and magnetic NF estimated cartographies are compared with the reference distributions in a simplified configuration and in an experimental test. Obtained results are evaluated in order to discuss conventional GA and EMTR technique performances in terms of simplicity, computing time and accuracy.

\section{THEORETICAL INVESTIGATIONS}

Generally, EM Interferences (EMI) are generated by straight and loop currents that can be modeled as electric and magnetic dipoles, Fig. 1. The set of these equivalent dipoles, relevant to a Device Under Test (DUT), are able to generate the same radiation field as the real studied structure, as explained in Fig. 2. In the literature, analytical expressions of radiated field generated by the elemental dipoles are well known and can be represented in the FD as in [5-6] or in the $\mathrm{TD}$ as in [15-16]. The obtained behavioral equivalent model represents the EM radiation in the TD (Part A) or in the FD (Part B). For the scope of this paper, explored methods are based on magnetic radiated field using only magnetic dipoles. 
Indeed, each obtained equivalent dipole is characterized by six parameters, which are: the coordinates of the equivalent dipole center $\left(X_{d}, Y_{d}, Z_{d}\right)$, angles of orientations $\left(\theta_{d}, \varphi_{d}\right)$ of the dipole moment, and the magnitude of the dipole moment $\left(M_{d}=I_{0} \times S\right.$ for a magnetic dipole, where $I_{0}$ is the current in the dipole, and $\mathrm{S}$ is the surface of the loop).

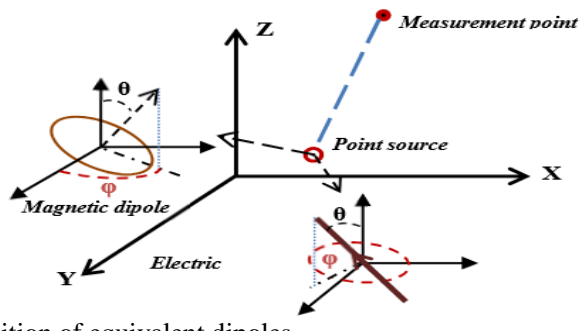

Fig. 1. Definition of equivalent dipoles.

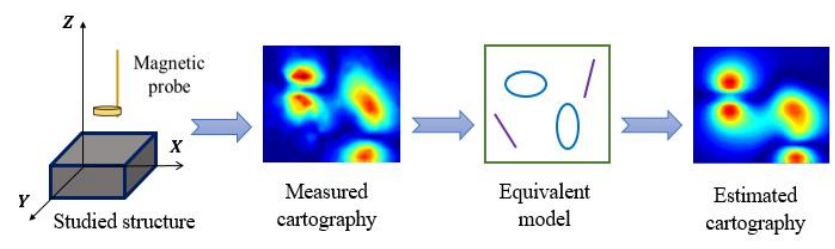

Fig. 2. Principle of EM inverse problem resolution.

\section{A. Electromagnetic inverse method based on EMTR}

The application of the EMTR technique in order to identify radiating sources for transient disturbances characterization particularly in the EM compatibility context is explained in [15-16]. In fact, the prediction of an equivalent radiation model of a DUT based on EMTR technique is an accurate and efficient procedure.

The basic idea of the electromagnetic time reversal technique is to take advantage of the reversibility in time of the wave propagation equation [11-16]. Indeed the EMTRbased method has the following main steps, Fig. 3:

1) Measurement or computation of originated EM transients at Time Reversal Mirror (TRM), which is a set of receiving antennas that record radiated magnetic field. This is the forward propagation phase.

2) Time reversing of recorded magnetic field and simulation of the back-injected signals. This is the time reversing phase.

3) Propagation of time-reversed signals. This is the backpropagation phase.

4) Assessment of obtained focusing results by determining, in a network domain, the most characterized source by the largest energy concentration. Indeed, time-reversed backpropagated field signals combine constructively to reach a maximum at the correct radiating source position. This is the focusing phase.

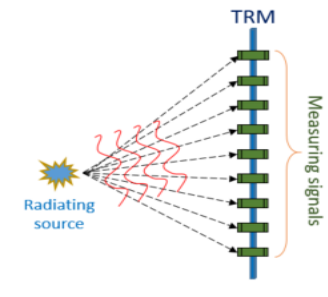

Propagation $\rightarrow$

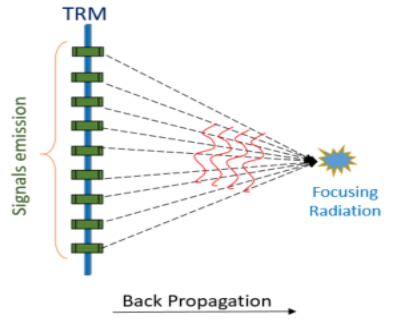

Back Propagation,
Fig. 3. Principle of EM Time Reversal technique.
It is worth noting that time-reversed radiated field signals converge, at the focusing time, to a specified space location, Fig. 3. This obtained position corresponds to the real radiating source. Moreover, the initial moment signal matches with the reversed focused signal at the obtained source location.

\section{B. Electromagnetic inverse method based on GA}

To better evaluate the EMTR technique, the proposed approach is compared to a standard GA-based method that has shown good performance in solving global optimization problems for radiating sources identification issue. The genetic algorithms are established on optimally modifying equivalent dipole parameters in order to minimize the error threshold described by the fitness function, which is defined as the difference between the reference field cartography and the estimated distribution.

The flowchart of the EM inverse method based on GA is illustrated in Fig. 4. To the aim of this comparative study, magnetic field signals have been obtained in the frequency domain. In order to accelerate the convergence of this process, a judicious choice of the parameters of the GA method has to be done [5]. Table I shows the chosen parameters of the GA.

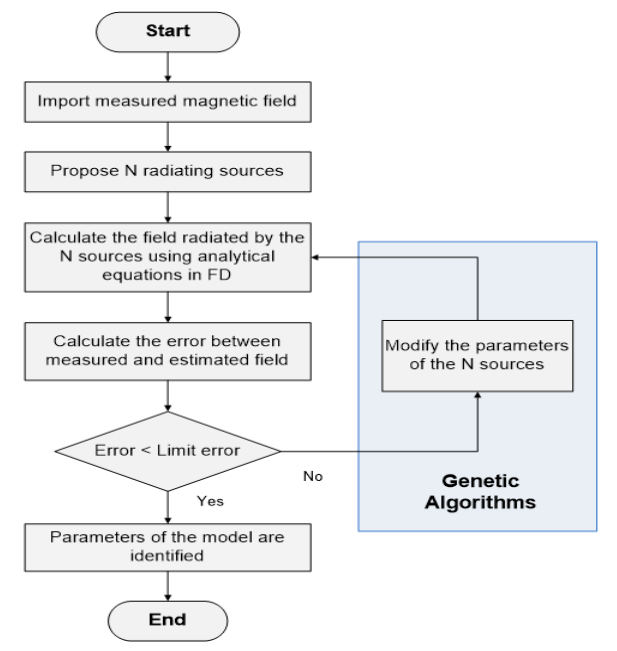

Fig. 4. Flowchart of EM inverse method based on GA.

TABLE I

ADOPTED GA PARAMETERS

\begin{tabular}{c|c}
\hline \hline Fitness Function & $\sum_{i=0}^{N} \frac{\left|H_{z_{\text {Reference }}}-H_{z_{\text {Estimated }}}\right|}{H_{z_{\text {Refrence }}}}$ \\
\hline Population size & $20 \times N_{\text {parameters }}<N_{p}<30 \times N_{\text {parameters }}$ \\
\hline Selection function & Roulette \\
\hline Crossover rate & Between 0.6 and 0.8 \\
\hline \hline
\end{tabular}

\section{SIMULATION AND EXPERIMENTAL COMPARAISON}

In the section below, we apply separately the proposed EMTR-based technique and the GA-based method previously presented on the same studied structure. Furthermore, in this work, only the vertical component of the magnetic field is employed.

\section{A. Simulation test}

In the present study, a comparison between, a TD-based and FD-based method is presented. Indeed, in power electronics, devices emit important non-sinusoidal EM 
interferences of short duration with a wide frequency spectrum. To simplify this study, we choose to limit this simulation test for a will-known signal. As we know, a signal can be decomposed as a combination of different sine functions. The signal frequency components are identified using the Fourier transform. Therefore, to perform a comparison the same input data is used. FD and TD magnetic filed distributions are calculated using a specified moment signal. This signal is the sum of five periodic signals with different frequencies. Components frequencies are $f=$ $\{4 ; 8 ; 12 ; 24 ; 32\} \mathrm{MHz}$. In fact, FD calculations were carried out using analytical expressions of magnetic field, as defined in [5]. For the TD-based method, equations in [15-16] were adopted.

The studied structure has $(200 \mathrm{~mm} \times 200 \mathrm{~mm})$ dimensions. Magnetic field calculations were performed at a height of $3 \mathrm{~cm}$ above the DUT and with a displacement step of $4 \mathrm{~mm}$. A current loop of a radius $r=5 \mathrm{~mm}$ have the magnetic moment shown in Fig.5.a and of $T=0.5 \mu \mathrm{s}$ duration. Its corresponding spectrum is presented in Fig.5.b, where we can obviously observe the fives frequency components.

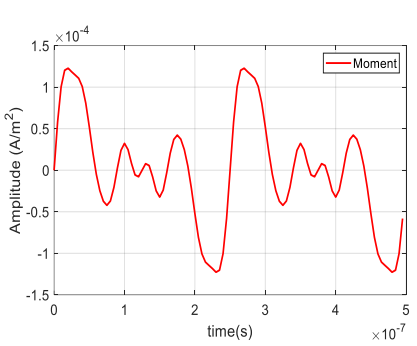

a) Moment

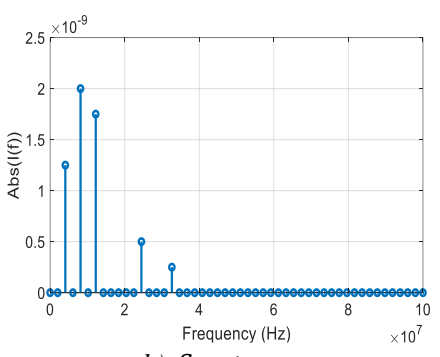

b) Spectrum
Fig. 5. Moment signal and its corresponding spectrum.

For the frequency method, the three-dimentional magnetic field components $H_{z}, H_{y}$ and $H_{x}$ are obtained for each single frequency component separetealy. For instance, field cartographies are presented in Fig. 6 for $f_{5}=32 \mathrm{MHz}$. Then, the genetic algorithm is applied for each frequency, as explained in Fig.4. The performance of this method is presented in Table II.
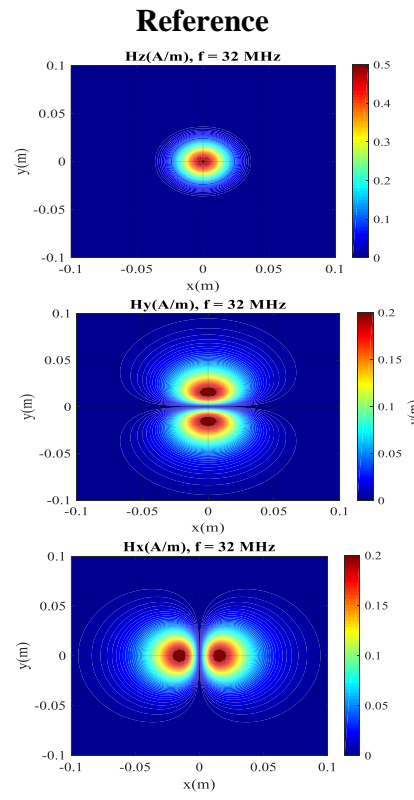
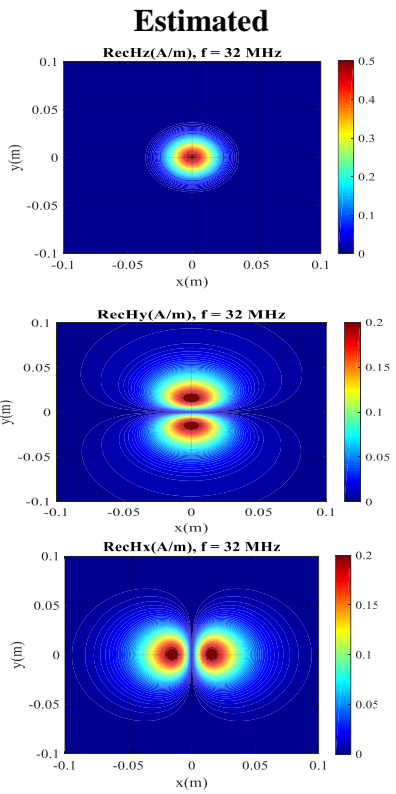

Fig. 6. Comparison of FD cartographies at $f_{5}=32 \mathrm{MHz}$.
TABLE II

GA METHOD PERFORMANCE

\begin{tabular}{c|c|c|c|c}
\hline \hline Sources & $\begin{array}{c}\text { Frequency } \\
(\mathbf{M H z})\end{array}$ & Generation & $\begin{array}{c}\text { Fitness } \\
(\boldsymbol{\%})\end{array}$ & $\begin{array}{c}\text { Computing time } \\
(\sim \text { min })\end{array}$ \\
\hline $\mathbf{1}$ & 4 & 292 & 2.75 & 31 \\
\hline $\mathbf{2}$ & 8 & 293 & 2.98 & 23 \\
\hline $\mathbf{3}$ & 12 & 956 & 2.00 & 53 \\
\hline $\mathbf{4}$ & 24 & 900 & 2.99 & 40 \\
\hline $\mathbf{5}$ & 32 & 4872 & 2.94 & 113 \\
\hline \hline
\end{tabular}

Although the classic GA-based method gives a correct field estimation, an iterative process has to be performed for every single frequency of the spectrum. Contrariwise, the TD-based method treats the whole wave propagation film. The magnetic field is calculated at each step time. Thus, the characterization process is obtained all at once. The processing time of the EMTR method is about $9 \mathrm{~min}$. Magnetic field cartographies are visualized, in Fig.7, at the time of the maximum magnitude occurrence. A satisfactory agreement is observed between the reference distribution and the estimated cartographies. In addition, a good estimation is obtained in Fig. 8 between original and reconstructed moment signals.
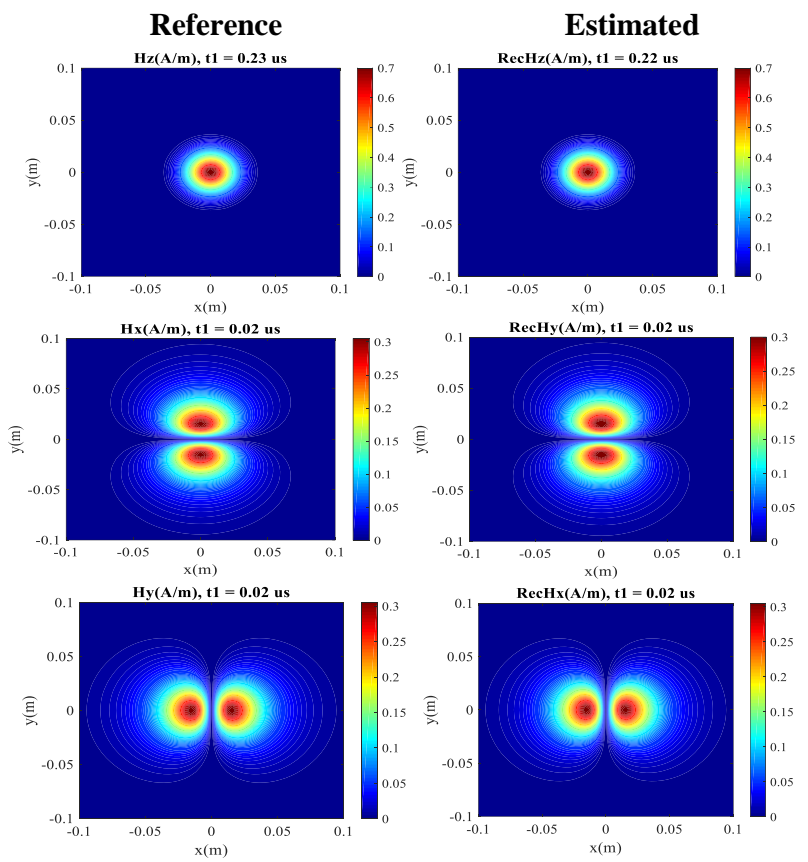

Fig. 7. Comparison of TD cartographies: $H_{z}$ at $t=0,22 u s$ and $H_{x}$ and $H_{y}$ at $t=0,02 u s$.

TABLE III

OBTAINED DIPOLE PARAMETERS

\begin{tabular}{c|c|c|c}
\hline \hline \multirow{3}{*}{ Method } & $\begin{array}{c}\text { Moment } \\
M_{d} \\
\left(A / m^{2}\right) \times e^{-4}\end{array}$ & $\begin{array}{c}\text { Position } \\
X_{d}, Y_{d}, Z_{d} \\
(\mathrm{~mm}) \times e^{-4}\end{array}$ & $\begin{array}{c}\text { Orientation } \\
\theta_{d}, \varphi_{d} \\
(\mathrm{rad})\end{array}$ \\
\hline EMTR & 1.22 & $0 ; 0 ; 0$ & $0 ; 0$ \\
\hline $\boldsymbol{G A}$ & 1.2 & $0.012 ; 7 ; 0.086$ & 0,$0013 ; 6,26$ \\
\hline \hline
\end{tabular}

The identified structure is composed of a magnetic dipole excited by a transient signal. Obtained parameters are summarized in Table III for the two approaches. A good estimation is performed by both methods. Since the GAbased method needs to be performed at each frequency component of the spectrum, whereas the EMTR-based 
method is carried out all at once, we can conclude that the EMTR method is much more straightforward, Table IV. Moreover, in term of processing time and due to the GA iterative process, the EMTR technique allows reducing the computational time by about $96 \%$.

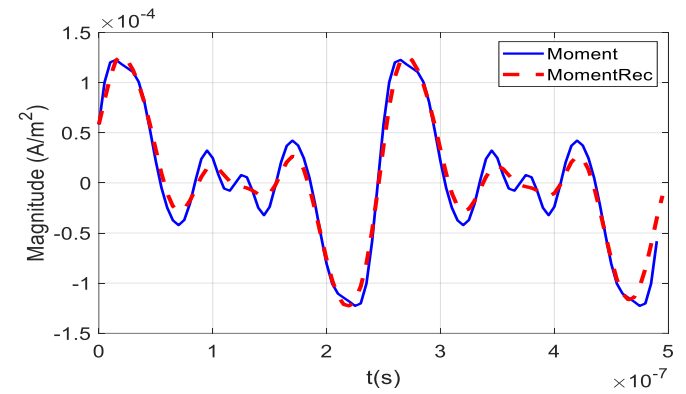

Fig. 8. Moment reconstruction.

TABLE IV

PERFORMANCE OF EACH METHOD

\begin{tabular}{l|c|c}
\hline \hline & $\begin{array}{c}\text { EMTR-based } \\
\text { method }\end{array}$ & GA-based method \\
\hline Model Simplicity & ++ & + \\
\hline $\begin{array}{c}\text { Computing time } \\
(\text { In } \text { min })\end{array}$ & 9.2 & 260 \\
\hline \hline
\end{tabular}

\section{B. Experimental test}

In this section, we compare the two proposed methods with an experimental measurement process above a simple structure, Fig.9. The studied PCB, of surface $(21 \mathrm{~mm} \times$ $21 \mathrm{~mm}$ ), is a magnetic loop, of $5 \mathrm{~mm}$ radius, carrying a non-sinusoidal current out coming from a chopper circuit that provides a high current and leads to severe EM interference issues especially due to the switching of transistor, Fig.9. Measurements are performed using the near field scanning technique, at a height of $8.5 \mathrm{~mm}$. Radiated fields are captured by a magnetic field probe that moves above the DUT with a $1.5 \mathrm{~mm}$ resolution. The NF measurement of the magnetic field emissions are carried out. Thereafter, both methods are applied in order to find an equivalent radiation model for this mono turn coil. Consequently, the two obtained behavioural models are compared.

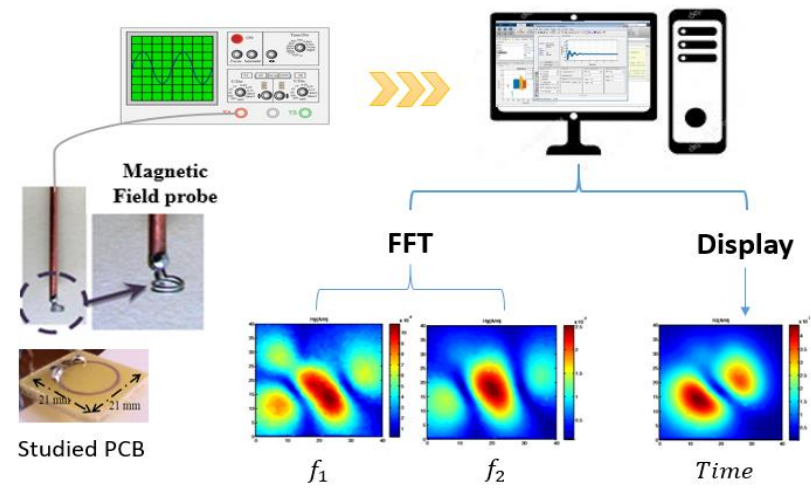

Fig. 9. Near-field measurement test bench.

The fast Fourier transform is applied to the different measured magnetic field signals. Looking at Fig. 10, in the frequency spectrum, we notice the appearance of several harmonics over a large frequency range that covers tens of megahertz. Accordingly, in order to obtain an equivalent model that perform the same EM disturbances as the studied PCB using the frequency inverse method based on the conventional GA, it is necessary to search a valid radiation pattern for each single frequency. However, this requires a considerable computation time. Fig. 11 shows measured $H_{z}$ magnetic field distributions for four different frequencies.

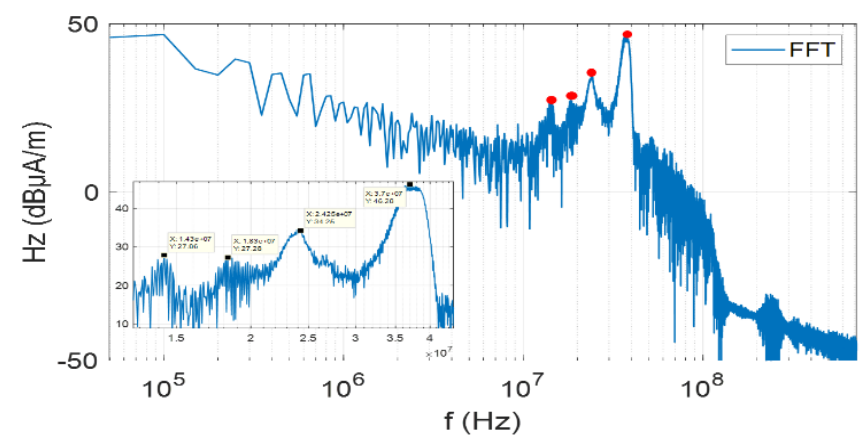

Fig. 10. Magnetic field spectrum measured above the studied structure.
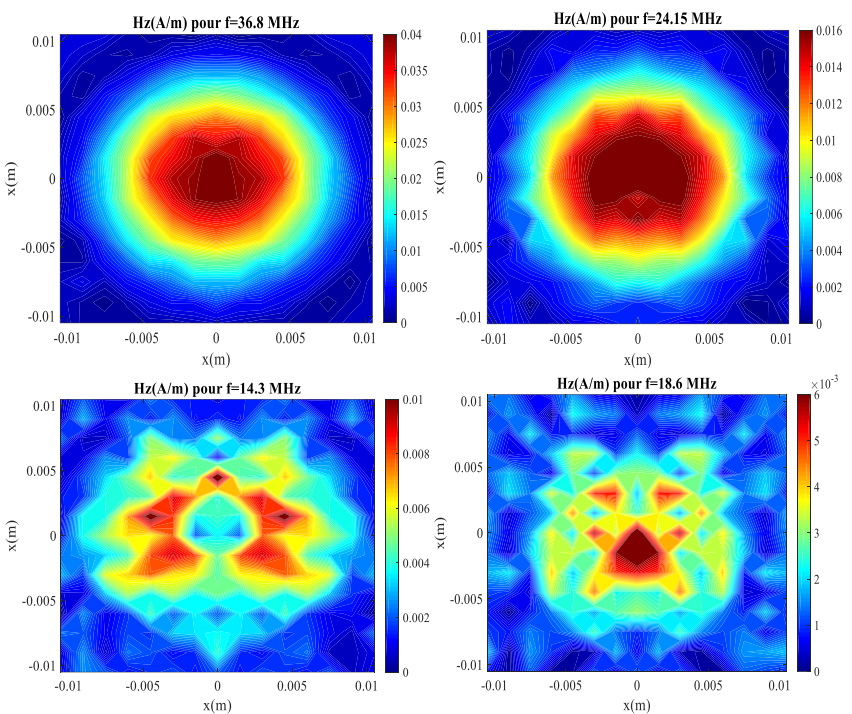

Fig. 11. Measured $H_{z}$ cartographies at $f_{1}=36.8 \mathrm{MHz}, f_{2}=$ 24.15 $\mathrm{MHz}$, at $f_{3}=14.3 \mathrm{MHz}$ at $f_{4}=18.6 \mathrm{MHz}$.

The transient waveforms of the vertical magnetic field during the switching of the transistor are presented in Fig.12 for different measurements positions. The radiated TD field cartographies of the component $H_{z}$ are visualized in Fig.13 at different time steps. An additional benefit of TD modelling is the possibility of identifying the moment signal of the radiating source, as presented in Fig.14. Furthermore, Fig.15.b shows a comparison between estimated and measured cuts of the normal component $\mathrm{Hz}$ along the $x$ axes $t=10.12 \mu \mathrm{s}$.

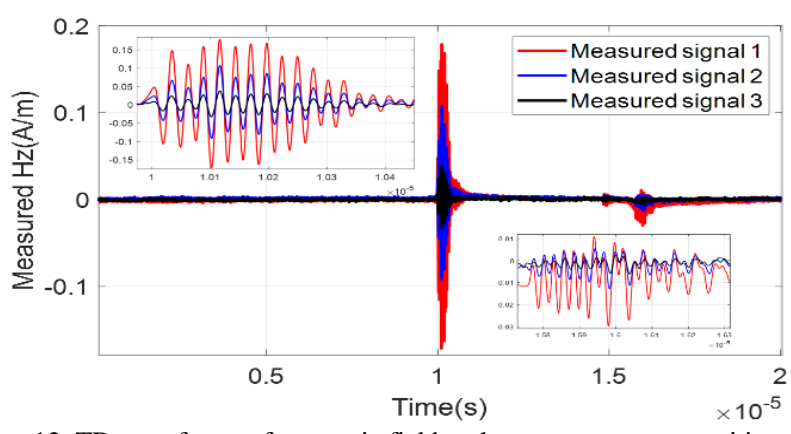

Fig. 12. TD waveforms of magnetic field at three measurement positions. 

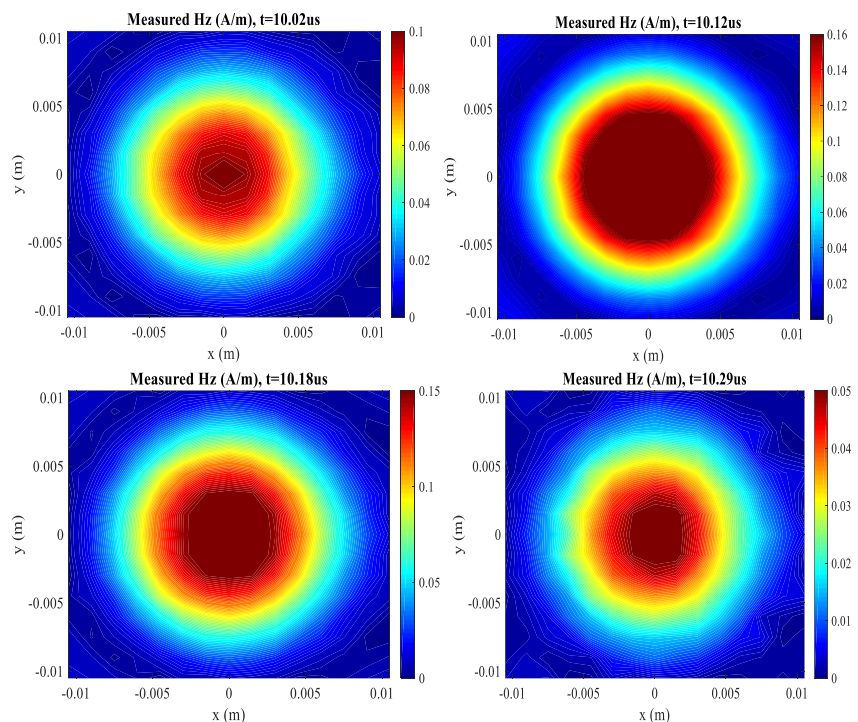

Fig. 13. Magnetic field cartographies $H_{z}$ at: $t_{1}=10.02 \mu \mathrm{s}, t_{2}=$ $10.12 \mu s, t_{3}=10.18 \mu s$ and $t_{4}=10.29 \mu s$.

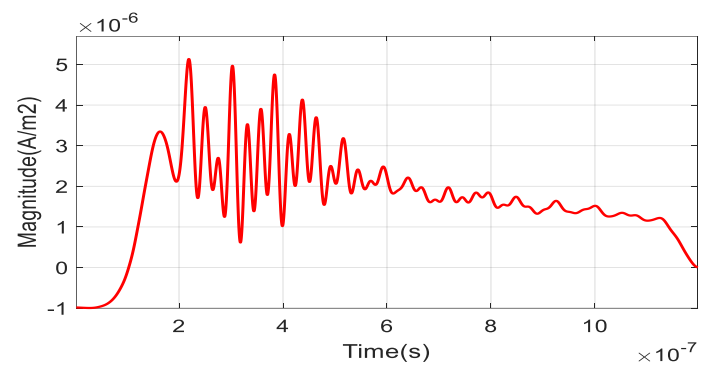

Fig. 14. Moment signal reconstruction.
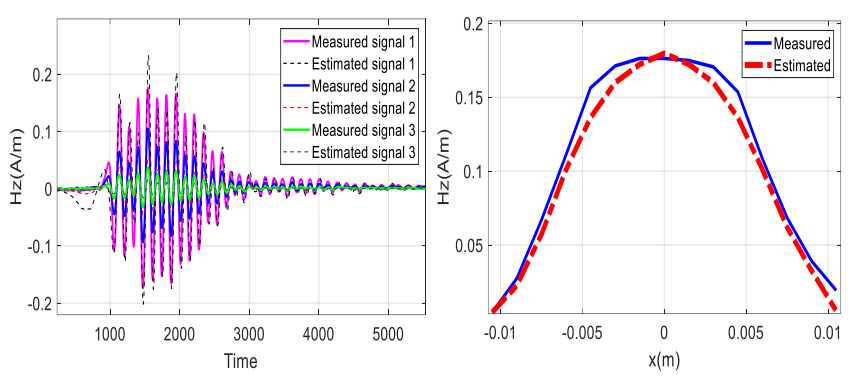

a) $H_{z}$ Field

b) Cut at $Y=0$ and $t=10.12 \mu \mathrm{s}$

Fig. 15. Radiated field reconstruction (Measured and estimated).
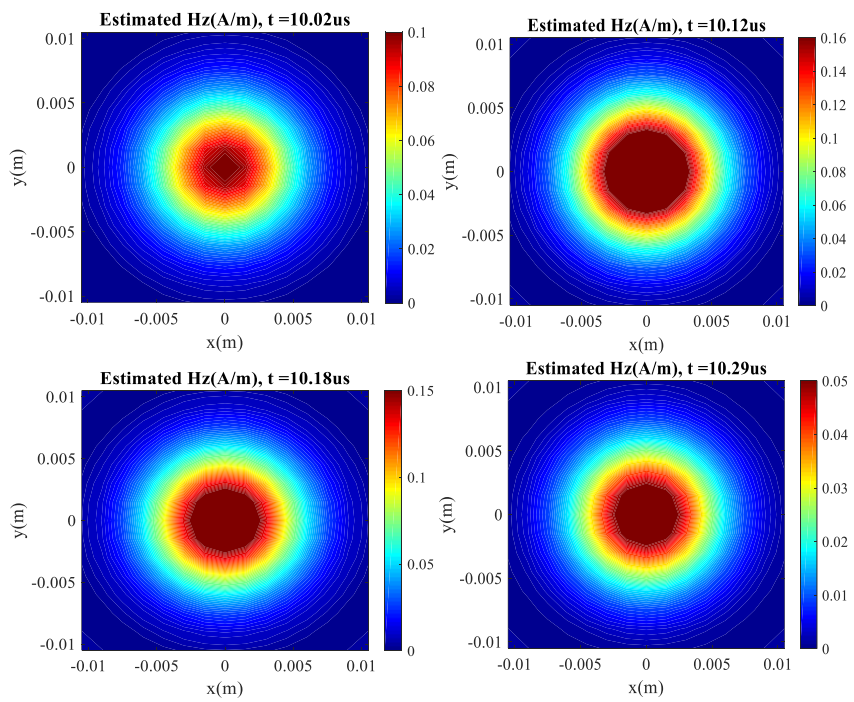

Fig. 16. Estimated field cartographies $H_{z}$ at: $t_{1}=10.02 \mu s, t_{2}=$ $10.12 \mu \mathrm{s}, t_{3}=10.18 \mu \mathrm{s}$ and $t_{4}=10.029 \mu \mathrm{s}$.
The identified structures using TD and FD methods are composed of a single magnetic loop that corresponds to the experimental studied PCB. The obtained parameters confirm the efficiency of the both methods, Table V. This can be further asserted by looking at Figs. 15-18. Indeed, Fig.17 presents the obtained estimation of the measured radiated fields at $f_{1}=36.8 \mathrm{MHz}, f_{2}=24.15 \mathrm{MHz}$. Furthermore, Fig.18 shows cuts of radiated estimated and measured distributions at $Y=0$ for two different frequencies.
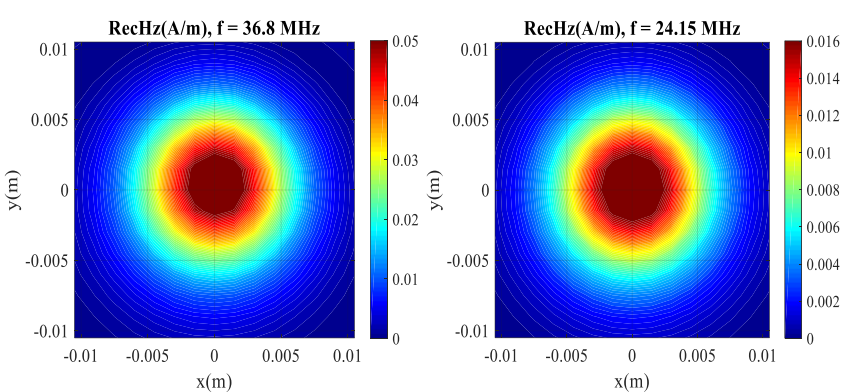

Fig. 17. Estimated FD cartographies at $f_{1}=36.8 \mathrm{MHz}, f_{2}=24.15 \mathrm{MHz}$.
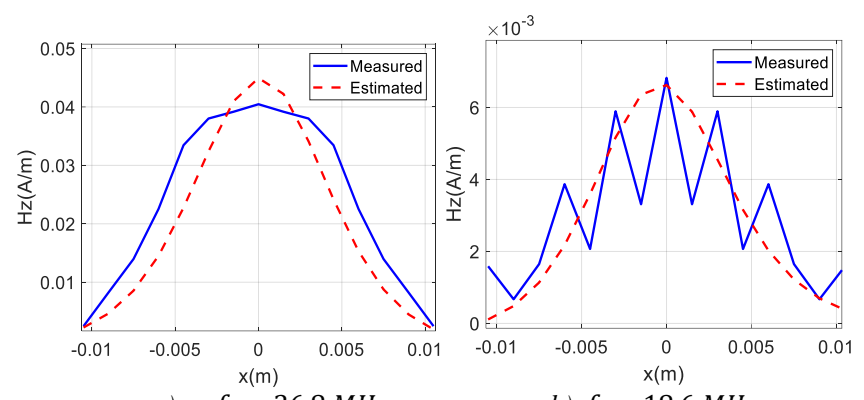

a) $f_{1}=36.8 \mathrm{MHz}$

Fig. 18. Field reconstruction: Cuts at $Y=0$.

TABLE V

OBTAINED DIPOLE PARAMETERS

\begin{tabular}{c|c|c|c|c}
\hline \hline \multirow{2}{*}{ Method } & $\begin{array}{c}\text { Moment } \\
M_{d} \\
\left(A / m^{2}\right) \times e^{-6}\end{array}$ & $\begin{array}{c}\text { Position } \\
X_{d}, Y_{d}, Z_{d} \\
(\mathrm{~mm}) \times e^{-4}\end{array}$ & $\begin{array}{c}\text { Orientation } \\
\theta_{d}, \varphi_{d} \\
(\mathrm{rad})\end{array}$ \\
\hline \multirow{2}{*}{ EMTR } & 5.14 & $0 ; 0 ; 0$ & $0 ; 0$ \\
\hline \multirow{2}{*}{$\boldsymbol{A}$} & $f_{1}$ & 0.417 & $2.73 ; 7.43 ;-2.5$ & $0.373 ; 6.13$ \\
\cline { 2 - 5 } & $f_{2}$ & 0.238 & $0.42 ; 4.65 ;-5$ & $3.17 ; 3.25$ \\
\hline \hline
\end{tabular}

To quantify the difference between the TD and FD methods, we propose to compare their computing time. Table VI presents a performance comparison between the two proposed approaches. Indeed, the processing time of the FD inverse method has to be performed particularly for the different resonance frequencies present in the spectrum (we have identified four resonance frequencies, Fig.10). Indeed, it corresponds to frequencies where the signal has high values. Therefore, the total processing time is calculated as the product of the obtained frequencies and the average computing time required to acquire the equivalent radiation model at a single frequency, knowing that only a single frequency, for instance $f_{1}=36.8 \mathrm{MHz}$, takes about $56 \mathrm{~min}$ to be processed.

Based on Table VI, we notice that the proposed EMTR technique allows minimizing computing time by about $64.04 \%$ compared to the classical frequency inverse method based on GA. Hence, this is a real test case where the use of TD-based method is strongly required. The application of the proposed EMTR technique, in the TD, allows the 
identification of a valid behavioural model of the studied PCB for all spectrum frequencies at once, which avoids the repetition of the same search process using the classic GA.

TABLE VI

PERFORMANCE OF EACH METHOD

\begin{tabular}{l|c|c}
\hline \hline & EMTR-based method & GA-based method \\
\hline Model Simplicity & ++ & + \\
\hline $\begin{array}{c}\text { Computing time } \\
(\text { In } \text { min) }\end{array}$ & 80.65 & $56 \times 4=224$ \\
\hline \hline
\end{tabular}

Therefore, it is noteworthy that frequency investigation on EM radiations of power electronics is not sufficient to deal with EM transients phenomena, especially when disturbances sources behave as a short period pulse wave. Hence, it is a hard task for an FFT-based algorithm to characterize and identify radiating sources. To accelerate the convergence of the GA method, options where judiciously chosen such as population size and lower and upper variables bounds. Furthermore, we highlight that the FD method is carried out at each single frequency among the frequency range. This requires a considerable measurement time. However, the TD proposed method allows a full measurement acquisition in a single test without loss of data. According to the obtained results, the TD inverse method based on the EMTR technique seems to be an alternative method for the FD methods. The proposed approach provides an accurate equivalent model in a reasonable processing time.

\section{CONCLUSION}

This paper presents a comparative study between the time domain electromagnetic inverse method based on the electromagnetic time reversal technique and the frequency domain electromagnetic inverse problem based on genetic algorithms. Both methods are using the near field scanning technique. The aim of the two approaches is to determine an accurate and valid equivalent radiation model that performs the same emissions as the studied structure. In order to compare the proposed method and the commonly used GAbased method, we proposed to evaluate the radiated magnetic field. This comparison is carried out at three levels: model simplicity, processing time and the model accuracy. Both methods were applied to the same configurations in simulation and experimental test. Good agreement was presented between estimated and measured magnetic field distributions using the two methods. Obtained results have shown that the proposed EMTR technique can be a good alternative for the FD methods and that it provides an accurate equivalent behavioural pattern with a considerable reduction in computing time.

\section{REFERENCES}

[1] Y.Vives-Gilabert, C.Arcambal, A.Louis, F.deDaran, P.Eudeline, and B. Mazari, "Modeling magnetic radiations of electronic circuits using nearfieldscanningmethod,'IEEETrans.Electromagn.Compat.,vol.49,n o.2, pp. 391-400, May 2007.

[2] H. Shall, Z. Riah, and M. Kadi, "A 3D near-field modeling approach for electromagnetic prediction," IEEE Trans. Electromagn. Compat., vol. 56, no. 1, pp. 102-112, Feb. 2014

[3] L. Beghou, F. Costa and L. Pichon, "Detection of Electromagnetic Radiations Sources at the Switching Time Scale Using an Inverse Problem-Based Resolution Method-Application to Power Electronic Circuits," in IEEE Transactions on Electromagnetic Compatibility, vol. 57, no. 1, pp. 52-60, Feb. 2015.

[4] B. Liu, L. Beghou, and L. Pichon, "Adaptive genetic algorithm based source identification with near-field scanning method," Progress Electromagn. Res. B, vol. 9, pp. 215-230, 2008.

[5] S. Saidi and J. Ben Hadj Slama, "Effect of Genetic Algorithm parameters on convergence of the electromagnetic inverse method," Eighth International Multi-Conference on Systems, Signals \& Devices, Sousse, 2011, pp. 1-5.S.

[6] S. Saidi and J. Ben Hadj Slama, "A Near-Field Technique Based on PZMI, GA, and ANN: Application to Power Electronics Systems," in IEEE Transactions on Electromagnetic Compatibility, vol. 56, no. 4, pp. 784-791, Aug. 2014

[7] W. Zhao et al., "An Effective and Efficient Approach for Radiated Emission Prediction Based on Amplitude-Only Near-Field Measurements," in IEEE Transactions on Electromagnetic Compatibility, vol. 54, no. 5, pp. 1186-1189, Oct. 2012.

[8] Y. Liu, B. Ravelo and A. K. Jastrzebski, "Time-Domain Magnetic Dipole Model of PCB Near-Field Emission," in IEEE Transactions on Electromagnetic Compatibility, vol. 58, no. 5, pp. 1561-1569, Oct. 2016.

[9] A. K. Jastrzebski, Y. Liu and B. Ravelo, "Characterisation and modelling of near-field radiated emissions in the time-domain," 2012 Asia-Pacific Symposium on Electromagnetic Compatibility, Singapore, 2012, pp. 201-204.

[10] B. Zitouna and J. Ben Hadj Slama, "Enhancement of Time-Domain Electromagnetic Inverse Method for Modeling Circuits Radiations," in IEEE Transactions on Electromagnetic Compatibility, vol. 58, no. 2, pp. 534-542, April 2016.

[11] F. Rachidi, M. Rubinstein and M. Paolone, Electromagnetic Time Reversal: Application to Electromagnetic Compatibility and Power Systems, Wiley, 2017

[12] A. Orlandi, "Electromagnetic Time Reversal Approach to Locate Multiple Soft Faults," in IEEE Transactions on Electromagnetic Compatibility, vol. 60, no. 4, pp. 1010-1013, Aug. 2018.

[13] Z. Wang, A. Codino, R. Razzaghi, M. Paolone and F. Rachidi, "Using electromagnetic time reversal to locate faults in transmission lines: Definition and application of the "Mirrored Minimum Energy" property," 2017 International Symposium on Electromagnetic Compatibility - EMC EUROPE, Angers, 2017, pp. 1-6.

[14] I. El Baba, S. Lallechere and P. Bonnet, "Electromagnetic TimeReversal for reverberation chamber applications using FDTD," 2009 International Conference on Advances in Computational Tools for Engineering Applications, Zouk Mosbeh, 2009, pp. 157-162.

[15] S. Hedia, B. Zitouna, J. Ben Hadj Slama and L. Pichon, "Electromagnetic Time Reversal for Radiating Source Identification in Time Domain," 2018 15th International Multi-Conference on Systems, Signals \& Devices (SSD), Yassmine Hammamet, Tunisia, 2018, pp. 531-536.

[16] S. Hedia, B. Zitouna, J. Ben Hadj Slama and L. Pichon, "A full time domain methodology based on near field time reversal for equivalent source identification," 2018 IEEE International Symposium on Electromagnetic Compatibility and 2018 IEEE Asia-Pacific Symposium on Electromagnetic Compatibility (EMC/APEMC), Singapore, 2018, pp. 141-146. 